\title{
On existence and essential stability of solutions of symmetric variational relation problems
}

\section{Zhe Yang*}

\section{"Correspondence:}

zheyang211@163.com

School of Economics, Shanghai

University of Finance and

Economics, Shanghai, 200433,

China

Key Laboratory of Mathematical Economics (SUFE), Ministry of

Education, Shanghai, 200433, China

\begin{abstract}
In this paper, we introduce symmetric variational relation problems and establish the existence theorem of solutions of symmetric variational relation problems. As the special cases, symmetric (vector) quasi-equilibrium problems and symmetric variational inclusion problems are obtained. Further, we study the notion of essential stability of equilibria of symmetric variational relation problems. We prove that most of symmetric variational relation problems (in the sense of Baire category) are essential and, for any symmetric variational relation problem, there exists at least one essential component of its solution set.
\end{abstract}

MSC: 49J53; 49J40

Keywords: symmetric variational relation problems; existence; essential stability

\section{Introduction}

Let $X$ and $Y$ be real locally convex Hausdorff spaces, and let $C$ and $D$ be nonempty subsets of $X$ and $Y$, respectively. Let $S: C \times D \rightrightarrows C$ and $T: C \times D \rightrightarrows D$ be set-valued mappings, and $f, g: C \times D \longrightarrow R$ be real functions. According to Noor and Oettli [1], the symmetric quasiequilibrium problem (SQEP) consists in finding $\left(x^{*}, y^{*}\right) \in C \times D$ such that $x^{*} \in S\left(x^{*}, y^{*}\right)$, $y^{*} \in T\left(x^{*}, y^{*}\right)$, and

$$
\begin{aligned}
& f\left(x, y^{*}\right) \geq f\left(x^{*}, y^{*}\right), \quad \forall x \in S\left(x^{*}, y^{*}\right), \\
& g\left(x^{*}, y\right) \geq g\left(x^{*}, y^{*}\right), \quad \forall y \in T\left(x^{*}, y^{*}\right) .
\end{aligned}
$$

The problem is a generalization of equilibrium problem proposed by Blum and Oettli [2]. The equilibrium problem contains as special cases, for instance, optimization problems, problems of Nash equilibria, fixed point problems, variational inequalities, and complementarity problems.

$\mathrm{Fu}$ [3] introduced symmetric vector quasi-equilibrium problems (SVQEP). Let $Z$ be a real Hausdorff topological vector space, and let $P \subset Z$ be a closed convex, pointed cone with apex at the origin and with int $P \neq \emptyset$, where int $P$ denotes the interior of $P$. Let $X, Y$, $C, D, S, T$ be as above. Let vector mappings $f, g: C \times D \longrightarrow Z$ be given. The symmetric vector quasi-equilibrium problem (SVQEP) consists in finding $\left(x^{*}, y^{*}\right) \in C \times D$ such that

\section{Springer}

@2014 Yang; licensee Springer. This is an Open Access article distributed under the terms of the Creative Commons Attribution License (http://creativecommons.org/licenses/by/2.0), which permits unrestricted use, distribution, and reproduction in any medium, provided the original work is properly cited. 
$x^{*} \in S\left(x^{*}, y^{*}\right), y^{*} \in T\left(x^{*}, y^{*}\right)$, and

$$
\begin{array}{ll}
f\left(x, y^{*}\right)-f\left(x^{*}, y^{*}\right) \notin-\operatorname{int} P, & \forall x \in S\left(x^{*}, y^{*}\right), \\
g\left(x^{*}, y\right)-g\left(x^{*}, y^{*}\right) \notin-\operatorname{int} P, & \forall y \in T\left(x^{*}, y^{*}\right) .
\end{array}
$$

Farajzadeh [4] considered symmetric vector quasi-equilibrium problems in the Hausdorff topological vector space by means of a particular technique and answered an open question raised by $\mathrm{Fu}$. The stability of the set of solutions for symmetric vector quasiequilibrium problems is discussed in [5-7]. Fakhar and Zafarani [8] introduced generalized symmetric vector quasi-equilibrium problems (GSVQEP). Let $X, Y, C, D, S, T, f, g$ be as above. Let $P$ be the set-valued mapping from $Z$ to $Z$ such that for every $z \in Z, P(z)$ is a pointed, closed and convex cone of $Z$ with a nonempty interior int $P(z)$ and $\theta: X \rightarrow Z$, $\eta: Y \longrightarrow Z$. The generalized symmetric vector quasi-equilibrium problem (GSVQEP) consists in finding $\left(x^{*}, y^{*}\right) \in C \times D$ such that $x^{*} \in S\left(x^{*}, y^{*}\right), y^{*} \in T\left(x^{*}, y^{*}\right)$, and

$$
\begin{array}{ll}
f\left(x, y^{*}\right)-f\left(x^{*}, y^{*}\right) \notin-\operatorname{int} P\left(\eta\left(y^{*}\right)\right), & \forall x \in S\left(x^{*}, y^{*}\right), \\
g\left(x^{*}, y\right)-g\left(x^{*}, y^{*}\right) \notin-\operatorname{int} P\left(\theta\left(x^{*}\right)\right), & \forall y \in T\left(x^{*}, y^{*}\right) .
\end{array}
$$

It is well known that the equilibrium problems are unified models of several problems, namely, optimization problems, saddle point problems, variational inequalities, fixed point problems, Nash equilibrium problems etc. Recently, Luc [9] introduced a more general model of equilibrium problems, which is called a variational relation problem (in short, VR). The stability of the solution set of variational relation problems was studied in $[10,11]$. Further studies of variational relation problems have been done. Lin and Wang [12] studied simultaneous variational relation problems (SVR) and related applications. Balaj and Luc [13] introduced mixed variational relation problems (MR), and established existence of solutions to a general inclusion problem. Particular cases of variational inclusions and intersections of set-valued mappings were also discussed in [14]. Balaj and Lin [15] brought forward generalized variational relation problems (GVR), and obtained an existence theorem of the solutions for a variational relation problem. An existence theorem for a variational inclusion problem, a KKM theorem and an extension of the well-known Ky Fan inequality have been established as particular cases. Lin and Ansari [16] introduced a system of quasi-variational relations and established the existence of solutions of SQVP by means of the maximal element theorem for a family of multivalued mappings.

Agarwal et al. [17] presented a unified approach in studying the existence of solutions for two types of variational relation problems, which encompass several generalized equilibrium problems, variational inequalities and variational inclusions investigated in the recent literature. Balaj and Lin [18] established existence criteria for the solutions of two very general types of variational relation problems. Moreover, Luc et al. [19] established two main existence conditions for solutions of variational relation problems without convexity, and Pu and Yang [20-22] studied variational relation problems without the KKM property and on Hadamard manifolds.

Motivated and inspired by research works mentioned above, in this paper, we introduce symmetric variational relation problems, and study the existence and essential stability of solutions of symmetric variational relation problems. The results of this paper improve 
and generalize several known results on variational relation problems and symmetric (vector) quasi-equilibrium problems.

\section{Symmetric variational relation}

Let $X, Y$ be two nonempty, compact and convex subsets of two normed linear spaces, respectively. Let $S: X \times Y \rightrightarrows X$ and $T: X \times Y \rightrightarrows Y$ be set-valued mappings, and $R(x, y, u)$, $Q(x, y, v)$ be two relations linking $x, u \in X$ and $y, v \in Y$. The symmetric variational relation problem consists in finding $\left(x^{*}, y^{*}\right) \in X \times Y$ such that $x^{*} \in S\left(x^{*}, y^{*}\right), y^{*} \in T\left(x^{*}, y^{*}\right)$, and

$$
\begin{array}{ll}
R\left(x^{*}, y^{*}, x\right) \text { holds, } & \forall x \in S\left(x^{*}, y^{*}\right), \\
Q\left(x^{*}, y^{*}, y\right) \text { holds, } & \forall y \in T\left(x^{*}, y^{*}\right) .
\end{array}
$$

We recall first some known results concerning set-valued mappings for later use.

Lemma 2.1 (17.35, 5.35 of [23]) (i) Assume that $X$ is a topological space, $Y$ is a locally convex space, and a correspondence $f: X \rightrightarrows Y$ is upper semicontinuous at some point $x_{0} \in$ $X$. If the set cl $\left(c o f\left(x_{0}\right)\right)$ is compact, then the closed convex hull correspondence clcof $: X \rightrightarrows$ $Y$ is upper semicontinuous at $x_{0}$. (ii) In a completely metrizable locally convex space, the closed convex hull of a compact set is compact.

Lemma 2.2 (5.29 of [23]) Let $A_{1}, \ldots, A_{n}$ be nonempty and compact subsets of the Hausdorff topological linear space. Then co $\left(\bigcup_{i=1}^{n} A_{i}\right)$ is compact.

Lemma 2.3 Let $A$ be a nonempty and compact subset of the compact normed linear space $E$, and let $B \supset A$ be open in $E$. Then $c l c o A \subset c o B$.

Proof Since $B \supset A$ is open in $E, c o B$ is also open. Then, for any $x \in A$, there exists an open convex neighborhood $V(x)$ of $x$ such that $x \in V(x) \subset c l V(x) \subset \operatorname{coB}$. Since $A$ is nonempty and compact, there is a finite subset $\left\{x_{1}, \ldots, x_{n}\right\}$ of $A$ such that

$$
A \subset \bigcup_{i=1}^{n} V\left(x_{i}\right) \subset \bigcup_{i=1}^{n} \operatorname{cl} V\left(x_{i}\right) \subset \operatorname{coB} B
$$

which implies that

$$
\operatorname{coA} \subset \operatorname{co}\left(\bigcup_{i=1}^{n} \operatorname{clV}\left(x_{i}\right)\right) \subset \operatorname{coB} .
$$

Since $c l V\left(x_{i}\right)$ is compact for any $i \in\{1, \ldots, n\}$, by Lemma 2.2, $c o\left(\bigcup_{i=1}^{n} c l V\left(x_{i}\right)\right)$ is compact. Hence

$$
\operatorname{clco} A \subset \operatorname{clco}\left(\bigcup_{i=1}^{n} \operatorname{cl} V\left(x_{i}\right)\right)=\operatorname{co}\left(\bigcup_{i=1}^{n} \operatorname{cl} V\left(x_{i}\right)\right) \subset \operatorname{coB} .
$$

The following theorem is the main result of this paper.

Theorem 2.1 Assume that

(i) $X, Y$ are two nonempty, compact and convex subsets of two normed linear spaces;

(ii) $S, T$ are continuous with nonempty convex compact values; 
(iii) $R(\cdot, \cdot, \cdot)$ and $Q(\cdot, \cdot, \cdot)$ are closed;

(iv) for any $y \in Y$, any finite subset $\left\{x_{1}, \ldots, x_{n}\right\}$ of $X$ and any $x \in \operatorname{co}\left\{x_{1}, \ldots, x_{n}\right\}$, there is $i \in\{1, \ldots, n\}$ such that $R\left(x, y, x_{i}\right)$ holds;

(v) for any $x \in X$, any finite subset $\left\{y_{1}, \ldots, y_{n}\right\}$ of $Y$ and any $y \in \operatorname{co}\left\{y_{1}, \ldots, y_{n}\right\}$, there is $i \in\{1, \ldots, n\}$ such that $Q\left(x, y, y_{i}\right)$ holds.

Then the symmetric variational relation problem has at least one solution.

Proof Firstly, denote

$$
\begin{aligned}
& \operatorname{Gr}(R)=\{(x, y, u) \in X \times Y \times X \mid R(x, y, u) \text { holds }\}, \\
& \operatorname{Gr}(Q)=\{(x, y, v) \in X \times Y \times Y \mid Q(x, y, v) \text { holds }\} .
\end{aligned}
$$

Then $\operatorname{Gr}(R)$ and $\operatorname{Gr}(Q)$ are closed in $X \times Y \times X$ and $X \times Y \times Y$, respectively. Next, define two mappings $f: X \times Y \times X \longrightarrow \mathbb{R}$ and $g: X \times Y \times Y \rightarrow \mathbb{R}$ by

$$
\begin{aligned}
& f(x, y, u)=d_{1}((x, y, u), G r(R)), \\
& g(x, y, v)=d_{2}((x, y, v), G r(Q)),
\end{aligned}
$$

where $d_{1}$ and $d_{2}$ are the distance on $X \times Y \times X$ and $X \times Y \times Y$, respectively. Then (i) $f, g$ are continuous; (ii) $f(x, y, u)=0$ if and only if $R(x, y, u)$ holds, and $g(x, y, v)=0$ if and only if $Q(x, y, v)$ holds; (iii) $f(x, y, u) \geq 0$ for any $(x, y, u) \in X \times Y \times X$, and $g(x, y, v) \geq 0$ for any $(x, y, v) \in X \times Y \times Y$.

Now, define the mappings $H: X \times Y \rightrightarrows X$ and $F: X \times Y \rightrightarrows Y$ by

$$
\begin{aligned}
& H(x, y)=\left\{u \in S(x, y) \mid f(x, y, u)=\max _{u^{\prime} \in S(x, y)} f\left(x, y, u^{\prime}\right)\right\}, \\
& F(x, y)=\left\{v \in Q(x, y) \mid g(x, y, v)=\max _{v^{\prime} \in Q(x, y)} g\left(x, y, v^{\prime}\right)\right\} .
\end{aligned}
$$

Since $S, T$ are continuous with nonempty convex compact values, and $f, g$ are continuous, it follows from the well-known Berge maximum theorem that $H$ and $F$ are upper semicontinuous with nonempty compact values. By Lemma 2.1, $c l c o H$ and $c l c o F$ are upper semicontinuous with nonempty convex compact values. Thus, define the mapping $\varphi: X \times Y \rightrightarrows X \times Y$ by

$$
\varphi(x, y)=\operatorname{clcoH}(x, y) \times \operatorname{clcoF}(x, y) .
$$

By the well-known Fan-Glicksberg fixed point theorem, there exists $\left(x^{*}, y^{*}\right) \in X \times Y$ such that $\left(x^{*}, y^{*}\right) \in \varphi\left(x^{*}, y^{*}\right)$, i.e.,

$$
\begin{aligned}
& x^{*} \in \operatorname{clcoH}\left(x^{*}, y^{*}\right) \subset S\left(x^{*}, y^{*}\right), \\
& y^{*} \in \operatorname{clcoF}\left(x^{*}, y^{*}\right) \subset T\left(x^{*}, y^{*}\right) .
\end{aligned}
$$

Suppose that the result is false, without loss of generality, there is $u_{0} \in S\left(x^{*}, y^{*}\right)$ such that $R\left(x^{*}, y^{*}, u_{0}\right)$ does not hold. Then $f\left(x^{*}, y^{*}, u_{0}\right)>0$. By the definition of $H$, we have

$$
f\left(x^{*}, y^{*}, u\right) \geq f\left(x^{*}, y^{*}, u_{0}\right)>0
$$


for any $u \in H\left(x^{*}, y^{*}\right)$, i.e.,

$$
H\left(x^{*}, y^{*}\right) \subset\left\{u \in X: R\left(x^{*}, y^{*}, u\right) \text { does not hold }\right\} .
$$

Since $R(\cdot, \cdot, \cdot)$ is closed, $\left\{u \in X: R\left(x^{*}, y^{*}, u\right)\right.$ does not hold $\}$ is open in $X$. By Lemma 2.3,

$$
\operatorname{clcoH}\left(x^{*}, y^{*}\right) \subset \operatorname{co}\left\{u \in X: R\left(x^{*}, y^{*}, u\right) \text { does not hold }\right\} \text {. }
$$

Since $x^{*} \in \operatorname{clcoH}\left(x^{*}, y^{*}\right), x^{*} \in \operatorname{co}\left\{u \in X: R\left(x^{*}, y^{*}, u\right)\right.$ does not hold $\}$, i.e., there is a finite subset $\left\{u_{1}, \ldots, u_{n}\right\} \subset\left\{u \in X: R\left(x^{*}, y^{*}, u\right)\right.$ does not hold $\}$ such that $x^{*} \in \operatorname{co}\left\{u_{1}, \ldots, u_{n}\right\}$. By condition (iv), there is $i_{0} \in\{1, \ldots, n\}$ such that $R\left(x^{*}, y^{*}, u_{i_{0}}\right)$ holds, which contradicts the fact that $R\left(x^{*}, y^{*}, u_{i}\right)$ does not hold for any $i \in\{1, \ldots, n\}$. This completes the proof.

Remark 2.1 By Theorem 2.1, we obtain the following typical examples.

(1) Let $f: X \times Y \longrightarrow \mathbb{R}$ and $g: X \times Y \rightarrow \mathbb{R}$ be two real-valued functions. Define the variational relations $R, Q$ as follows:

$$
\begin{aligned}
& R(x, y, u) \text { holds if and only if } f(x, y) \leq f(u, y) \\
& Q(x, y, v) \text { holds if and only if } g(x, y) \leq g(x, v) .
\end{aligned}
$$

The symmetric quasi-equilibrium problem (SQEP) consists in finding $\left(x^{*}, y^{*}\right) \in X \times Y$ such that $x^{*} \in S\left(x^{*}, y^{*}\right), y^{*} \in T\left(x^{*}, y^{*}\right)$, and

$$
\begin{aligned}
& f\left(x, y^{*}\right) \geq f\left(x^{*}, y^{*}\right), \quad \forall x \in S\left(x^{*}, y^{*}\right), \\
& g\left(x^{*}, y\right) \geq g\left(x^{*}, y^{*}\right), \quad \forall y \in T\left(x^{*}, y^{*}\right) .
\end{aligned}
$$

By virtue of Theorem 2.1, problem (SQEP) has at least one solution.

(2) Let $X, Y, S, T$ be as above, $Z$ be a real Hausdorff topological vector space, and $P \subset Z$ be a closed convex, pointed cone with apex at the origin and with int $P \neq \emptyset$, where int $P$ denotes the interior of $P$. Let vector mappings $f, g: C \times D \longrightarrow Z$ be given. Define the variational relations $R, Q$ as follows:

$$
\begin{aligned}
& R(x, y, u) \text { holds if and only if } f(u, y)-f(x, y) \notin-\operatorname{int} P \\
& Q(x, y, v) \text { holds if and only if } g(x, v)-g(x, y) \notin-\operatorname{int} P .
\end{aligned}
$$

The symmetric vector quasi-equilibrium problem (SVQEP) consists in finding $\left(x^{*}, y^{*}\right) \in$ $X \times Y$ such that $x^{*} \in S\left(x^{*}, y^{*}\right), y^{*} \in T\left(x^{*}, y^{*}\right)$, and

$$
\begin{aligned}
& f\left(x, y^{*}\right)-f\left(x^{*}, y^{*}\right) \notin-\operatorname{int} P, \quad \forall x \in S\left(x^{*}, y^{*}\right), \\
& g\left(x^{*}, y\right)-g\left(x^{*}, y^{*}\right) \notin-\operatorname{int} P, \quad \forall y \in T\left(x^{*}, y^{*}\right) .
\end{aligned}
$$

By virtue of Theorem 2.1, problem (SVQEP) has at least one solution.

(3) Let $X, Y, S, T$ be as above, $Z$ be a real Hausdorff topological vector space, $A, A^{\prime}$ : $X \times Y \times X \rightrightarrows Z$ and $B^{\prime}, B: X \times Y \times Y \rightrightarrows Z$ be two multivalued mappings. Define the 
variational relations $R, Q$ as follows:

$$
\begin{aligned}
& R(x, y, u) \text { holds if and only if } A(x, y, u) \subset B(x, y, u) \text {; } \\
& Q(x, y, v) \text { holds if and only if } A^{\prime}(x, y, v) \subset B^{\prime}(x, y, v) .
\end{aligned}
$$

The symmetric variational inclusion of type (I) consists in finding $\left(x^{*}, y^{*}\right) \in X \times Y$ such that $x^{*} \in S\left(x^{*}, y^{*}\right), y^{*} \in T\left(x^{*}, y^{*}\right)$, and

$$
\begin{array}{ll}
A\left(x^{*}, y^{*}, u\right) \subset B\left(x^{*}, y^{*}, u\right), & \forall u \in S\left(x^{*}, y^{*}\right), \\
A^{\prime}\left(x^{*}, y^{*}, v\right) \subset B^{\prime}\left(x^{*}, y^{*}, v\right), & \forall v \in T\left(x^{*}, y^{*}\right) .
\end{array}
$$

By virtue of Theorem 2.1, the symmetric variational inclusion of type (I) has at least one solution.

(4) Define the variational relations $R, Q$ as follows:

$$
\begin{aligned}
& R(x, y, u) \text { holds if and only if } A(x, y, u) \cap B(x, y, u) \neq \emptyset ; \\
& Q(x, y, v) \text { holds if and only if } A^{\prime}(x, y, v) \cap B^{\prime}(x, y, v) \neq \emptyset .
\end{aligned}
$$

The symmetric variational inclusion of type (II) problem consists in finding $\left(x^{*}, y^{*}\right) \in X \times$ $Y$ such that $x^{*} \in S\left(x^{*}, y^{*}\right), y^{*} \in T\left(x^{*}, y^{*}\right)$, and

$$
\begin{array}{ll}
A\left(x^{*}, y^{*}, u\right) \cap B\left(x^{*}, y^{*}, u\right) \neq \emptyset, & \forall u \in S\left(x^{*}, y^{*}\right), \\
A^{\prime}\left(x^{*}, y^{*}, v\right) \cap B^{\prime}\left(x^{*}, y^{*}, v\right) \neq \emptyset, & \forall v \in T\left(x^{*}, y^{*}\right) .
\end{array}
$$

By virtue of Theorem 2.1, the symmetric variational inclusion of type (II) has at least one solution.

(5) Define the variational relations $R, Q$ as follows:

$$
\begin{aligned}
& R(x, y, u) \text { holds if and only if } \mathbf{0} \in A(x, y, u) ; \\
& Q(x, y, v) \text { holds if and only if } \mathbf{0} \in A^{\prime}(x, y, v) .
\end{aligned}
$$

The symmetric variational inclusion of type (III) problem consists in finding $\left(x^{*}, y^{*}\right) \in$ $X \times Y$ such that $x^{*} \in S\left(x^{*}, y^{*}\right), y^{*} \in T\left(x^{*}, y^{*}\right)$, and

$$
\begin{array}{ll}
\mathbf{0} \in A\left(x^{*}, y^{*}, u\right), & \forall u \in S\left(x^{*}, y^{*}\right), \\
\mathbf{0} \in A^{\prime}\left(x^{*}, y^{*}, v\right), & \forall v \in T\left(x^{*}, y^{*}\right) .
\end{array}
$$

By virtue of Theorem 2.1, the symmetric variational inclusion of type (III) has at least one solution.

\section{Essential stability}

Essential components play an important role in the study of stability. Now, let us start studying the essential stability of solution set of symmetric variational relation problems. First, denote by $\mathcal{M}$ the collection of symmetric variational relation problems such that 
all conditions of Theorem 2.1 hold. For each $q \in \mathcal{M}$, denote by $\Lambda(q)$ the solution set of $q$. Thus, a set-valued correspondence $\Lambda: \mathcal{M} \rightrightarrows X \times Y$ is well defined. To analyze the stability of $\Lambda(q)$ in $\mathcal{M}$, some topological structure in the collection $\mathcal{M}$ is also needed. For each $q, q^{\prime} \in \mathcal{M}$, define the distance on $\mathcal{M}$ by

$$
\begin{aligned}
\rho\left(q, q^{\prime}\right)= & \sup _{(x, y) \in X \times Y} h_{X}\left(S(x, y), S^{\prime}(x, y)\right)+\sup _{(x, y) \in X \times Y} h_{Y}\left(T(x, y), T^{\prime}(x, y)\right) \\
& +h\left(\operatorname{Gr}(R), G r\left(R^{\prime}\right)\right)+h^{\prime}\left(\operatorname{Gr}(Q), \operatorname{Gr}\left(Q^{\prime}\right)\right),
\end{aligned}
$$

where $h_{X}$ is the Hausdorff distance defined on $X, h_{Y}$ is the Hausdorff distance defined on $Y, h$ is the Hausdorff distance defined on $X \times Y \times X$, and $h^{\prime}$ is the Hausdorff distance defined on $X \times Y \times Y$. Clearly, $(\mathcal{M}, \rho)$ is a metric space.

Definition 3.1 Let $q \in \mathcal{M}$. An $(x, y) \in \Lambda(q)$ is said to be an essential point of $\Lambda(q)$ if, for any open neighborhood $N(x, y)$ of $(x, y)$ in $X \times Y$, there is a positive $\delta$ such that $N(x, y) \cap \Lambda\left(q^{\prime}\right) \neq$ $\emptyset$ for any $q^{\prime} \in \mathcal{M}$ with $\rho\left(q, q^{\prime}\right)<\delta . q$ is said to be essential if each $(x, y) \in \Lambda(q)$ is essential.

Definition 3.2 Let $q \in \mathcal{M}$. A nonempty closed subset $e(q)$ of $\Lambda(q)$ is said to be an essential set of $\Lambda(q)$ if, for any open set $U, e(q) \subset U$, there is a positive $\delta$ such that $U \cap \Lambda\left(q^{\prime}\right) \neq \emptyset$ for any $q^{\prime} \in \mathcal{M}$ with $\rho\left(q, q^{\prime}\right)<\delta$.

Definition 3.3 Let $q \in \mathcal{M}$. An essential subset $m(q) \subset \Lambda(q)$ is said to be a minimal essential set of $\Lambda(q)$ if it is a minimal element of the family of essential sets ordered by set inclusion. A component $C(q)$ is said to be an essential component of $\Lambda(q)$ if $C(q)$ is essential.

Remark 3.1 (1) It is easy to see that the problem $q \in \mathcal{M}$ is essential if and only if the mapping $\Lambda: \mathcal{M} \rightrightarrows X \times Y$ is lower semicontinuous at $q$. (2) For two closed $e_{1}(q) \subset e_{2}(q) \subset$ $\Lambda(q)$, if $e_{1}(q)$ is essential, then $e_{2}(q)$ is also essential.

First of all, let us introduce some mathematical tools for the following proof, which can be found in [23-26].

Lemma 3.1 ([23]) Let $X$ and $Y$ be two topological spaces with $Y$ compact. If $F$ is a closed set-valued mapping from $X$ to $Y$, then $F$ is upper semi-continuous.

Lemma $3.2([24])$ If $X, Y$ are two metric spaces, $X$ is complete and $F: X \rightrightarrows Y$ is upper semicontinuous with nonempty compact values, then the set of points, where $F$ is lower semicontinuous, is a dense residual set in $X$.

Lemma 3.3 ([25]) Let C, D be two nonempty, convex and compact subsets of linear normed space E. Then

$$
h(C, \lambda C+\mu D) \leq h(C, D),
$$

where $h$ is the Hausdorff distance defined on $E$, and $\lambda, \mu \geq 0, \lambda+\mu=1$. 
Lemma 3.4 ([26]) Let $(Y, \rho)$ be a metric space, $K_{1}$ and $K_{2}$ be two nonempty compact subsets of $Y, V_{1}$ and $V_{2}$ be two nonempty disjoint open subsets of $Y$. If $h\left(K_{1}, K_{2}\right)<\rho\left(V_{1}, V_{2}\right):=$ $\inf \left\{\rho(x, y) \mid x \in V_{1}, y \in V_{2}\right\}$, then

$$
h\left(K_{1},\left(K_{1} \backslash V_{2}\right) \cup\left(K_{2} \backslash V_{1}\right)\right) \leq h\left(K_{1}, K_{2}\right),
$$

where $h$ is the Hausdorff metric defined on $Y$.

Theorem $3.1(\mathcal{M}, \rho)$ is a complete metric space.

Proof Let $\left\{q^{n}\right\}_{n=1}^{\infty}$ be any Cauchy sequence in $\mathcal{M}$, then, for any $\varepsilon>0$, there is $N>0$ such that $\rho\left(q^{n}, q^{m}\right)<\varepsilon$ for any $n, m>N$, i.e.,

$$
\begin{aligned}
& \sup _{(x, y) \in X \times Y} h_{X}\left(S^{n}(x, y), S^{m}(x, y)\right)+\sup _{(x, y) \in X \times Y} h_{Y}\left(T^{n}(x, y), T^{m}(x, y)\right) \\
& +h\left(G r\left(R^{n}\right), G r\left(R^{m}\right)\right)+h^{\prime}\left(\operatorname{Gr}\left(Q^{n}\right), G r\left(Q^{m}\right)\right) \leq \varepsilon
\end{aligned}
$$

for any $n, m>N$.

(1) Clearly, there exist set-valued mappings $S: X \times Y \rightrightarrows X, T: X \times Y \rightrightarrows Y$, and a closed subset $A$ of $X \times Y \times X$, a closed subset $B$ of $X \times Y \times Y$ such that $S^{n}(x, y) \longrightarrow S(x, y)$, $T^{n}(x, y) \longrightarrow T(x, y)$ for any $(x, y) \in X \times Y$, and $S, T$ are continuous with nonempty convex compact values, and $\operatorname{Gr}\left(R^{n}\right) \longrightarrow A, \operatorname{Gr}\left(Q^{n}\right) \longrightarrow B$.

(2) Further, define the following symmetric variational relation $q=(S, T, R, Q)$ by

$$
\begin{aligned}
& R(x, y, u) \text { holds if and only if }(x, y, u) \in A, \\
& Q(x, y, v) \text { holds if and only if }(x, y, v) \in B .
\end{aligned}
$$

Clearly, $q^{n} \longrightarrow q$ under the distance $\rho$. We will show that $q \in \mathcal{M}$.

(i) Clearly, $R(\cdot, \cdot, \cdot)$ and $Q(\cdot, \cdot, \cdot)$ are closed.

(ii) Suppose that there exist $y \in Y$, a finite subset $\left\{x_{1}, \ldots, x_{n}\right\}$ of $X$ and $x \in \operatorname{co}\left\{x_{1}, \ldots, x_{n}\right\}$ such that $R\left(x, y, x_{i}\right)$ does not hold for each $i \in\{1, \ldots, n\}$, then $\left(x, y, x_{i}\right) \notin A$ for each $i \in$ $\{1, \ldots, n\}$. Since $q^{m} \longrightarrow q$, then $\left(x, y, x_{i}\right) \notin G r\left(R^{m}\right)$ for each $i \in\{1, \ldots, n\}$ and for enough large $m$, which implies that $R^{m}\left(x, y, x_{i}\right)$ does not hold for each $i \in\{1, \ldots, n\}$. It is a contradiction. Thus, for any $y \in Y$, any finite subset $\left\{x_{1}, \ldots, x_{n}\right\}$ of $X$ and any $x \in \operatorname{co}\left\{x_{1}, \ldots, x_{n}\right\}$, there is $i \in\{1, \ldots, n\}$ such that $R\left(x, y, x_{i}\right)$ holds. Similarly, for any $x \in X$, any finite subset $\left\{y_{1}, \ldots, y_{n}\right\}$ of $Y$ and any $y \in \operatorname{co}\left\{y_{1}, \ldots, y_{n}\right\}$, there is $i \in\{1, \ldots, n\}$ such that $Q\left(x, y, y_{i}\right)$ holds. Hence $q \in \mathcal{M}$ and $(\mathcal{M}, \rho)$ is complete.

Theorem 3.2 The solution mapping $\Lambda: \mathcal{M} \rightrightarrows X \times Y$ is upper semicontinuous with nonempty compact values.

Proof The desired conclusion follows from Lemma 3.1 as soon as we show that $\operatorname{Graph}(\Lambda)$ is closed. Let $\left\{\left(q^{n}, x^{n}, y^{n}\right) \in \mathcal{M} \times X \times Y\right\}_{n=1}^{\infty}$ be a sequence converging to $(q, x, y)$ such that $\left(x^{n}, y^{n}\right) \in \Lambda\left(q^{n}\right)$ for any $n$. Then $x^{n} \in S^{n}\left(x^{n}, y^{n}\right)$ and $y^{n} \in T^{n}\left(x^{n}, y^{n}\right), R^{n}\left(x^{n}, y^{n}, u\right)$ and 
$Q^{n}\left(x^{n}, y^{n}, v\right)$ hold for any $u \in S^{n}\left(x^{n}, y^{n}\right)$ and any $v \in T^{n}\left(x^{n}, y^{n}\right)$. Since $q^{n} \longrightarrow q$, it follows that $x \in S(x, y)$ and $y \in T(x, y)$.

Suppose that there exists $u_{0} \in S(x, y)$ such that $R\left(x, y, u_{0}\right)$ does not hold, then there exists $u^{n} \in S^{n}\left(x^{n}, y^{n}\right)$ such that $u^{n} \longrightarrow u_{0}$, and $\left(x, y, u_{0}\right) \notin G r(R)$. For enough large $n$, we have $\left(x^{n}, y^{n}, u^{n}\right) \notin \operatorname{Gr}\left(R^{n}\right)$, i.e., $R^{n}\left(x^{n}, y^{n}, u^{n}\right)$ does not hold. It is a contradiction. Therefore $R(x, y, u)$ holds for any $u \in S(x, y)$. Similarly, $Q(x, y, v)$ holds for any $v \in T(x, y)$. Hence $(x, y) \in \Lambda(q)$.

Theorem 3.3 There exists a dense residual subset $q$ of $\mathcal{M}$ such that $q$ is essential for each $q \in q$.

Proof Since the metric space $(\mathcal{M}, \rho)$ is complete (by Theorem 3.1), and the mapping $\Lambda: \mathcal{M} \rightrightarrows X \times Y$ is upper semicontinuous with compact values (by Theorem 3.2), by Lemma 3.2, $\Lambda$ is lower semicontinuous on a dense residual subset $g$ of $\mathcal{M}$. Thus, by Remark 3.1(1), $q$ is essential for each $q \in G$.

Theorem 3.4 For each $q \in \mathcal{M}$, there exists at least one minimal essential subset of $\Lambda(q)$.

Proof By Theorem 3.2, $\Lambda: \mathcal{M} \rightrightarrows X \times Y$ is upper semicontinuous with compact values, that is, for each open set $O \supset \Lambda(q)$, there exists $\delta>0$ such that $O \supset \Lambda\left(q^{\prime}\right)$ for any $q^{\prime} \in \mathcal{M}$ with $\rho\left(q, q^{\prime}\right)<\delta$. Hence $\Lambda(q)$ is an essential set of itself. Let $\Theta$ denote the family of all essential sets of $\Lambda(q)$ ordered by set inclusion. Then $\Theta$ is nonempty and every decreasing chain of elements in $\Theta$ has a lower bound (because by the compactness the intersection is in $\Theta$ ); therefore, by Zorn's lemma, $\Theta$ has a minimal element and it is a minimal essential set of $\Lambda(q)$.

Theorem 3.5 For each $q \in \mathcal{M}$, every minimal essential subset of $\Lambda(q)$ is connected.

Proof For each $q \in \mathcal{M}$, let $m(q) \subset \Lambda(q)$ be a minimal essential subset of $\Lambda(q)$. Suppose that $m(q)$ is not connected, then there exist two non-empty compact subsets $c_{1}(q), c_{2}(q)$ with $m(q)=c_{1}(q) \cup c_{2}(q)$, and there exist two disjoint open subsets $V_{1}, V_{2}$ of $X \times Y$ such that $V_{1} \supset c_{1}(q), V_{2} \supset c_{2}(q)$. Since $m(q)$ is a minimal essential set of $\Lambda(q)$, neither $c_{1}(q)$ nor $c_{2}(q)$ is essential. There exist two open sets $O_{1} \supset c_{1}(q), O_{2} \supset c_{2}(q)$ such that, for any $\delta>0$, there exist $q^{1}, q^{2} \in \mathcal{M}$ with

$$
\rho\left(q, q^{1}\right)<\delta, \quad \rho\left(q, q^{2}\right)<\delta, \quad \Lambda\left(q^{1}\right) \cap O_{1}=\emptyset, \quad \Lambda\left(q^{2}\right) \cap O_{2}=\emptyset .
$$

Denote $W_{1}=V_{1} \cap O_{1}, W_{2}=V_{2} \cap O_{2}$, we know that $W_{1}, W_{2}$ are open, $W_{1} \supset c_{1}(q), W_{2} \supset$ $c_{2}(q)$, and we may assume that $V_{1} \supset \bar{W}_{1}, V_{2} \supset \bar{W}_{2}$. Denote $G_{1}=W_{1} \times X$ and $G_{2}=W_{2} \times Y$, $\inf \left\{d(a, b) \mid x \in G_{1}, b \in G_{2}\right\}=\varepsilon>0$.

Since $m(q)$ is essential and $m(q) \subset\left(W_{1} \cup W_{2}\right)$, there exists $0<\delta^{*}<\varepsilon$ such that $\Lambda\left(q^{\prime}\right) \cap$ $\left(W_{1} \cup W_{2}\right) \neq \emptyset$ for any $q^{\prime} \in \mathcal{M}$ with $\rho\left(q, q^{\prime}\right)<\delta^{*}$. Since $m(q)$ is a minimal essential set of $\Lambda(q)$, neither $c_{1}(q)$ nor $c_{2}(q)$ is essential. Thus, for $\frac{\delta^{*}}{32}>0$, there exist two $q^{1}, q^{2} \in \mathcal{M}$ such that

$$
\Lambda\left(q^{1}\right) \cap W_{1}=\emptyset, \quad \Lambda\left(q^{2}\right) \cap W_{2}=\emptyset, \quad \rho\left(q^{1}, q\right)<\frac{\delta^{*}}{32}, \quad \rho\left(q^{2}, q\right)<\frac{\delta^{*}}{32} .
$$


Thus

$$
\rho\left(q^{1}, q^{2}\right)<\frac{\delta^{*}}{16}
$$

Next, define the symmetric variational relation problem $q^{\prime}=\left(S^{\prime}, T^{\prime}, R^{\prime}, Q^{\prime}\right)$ by

$$
\begin{aligned}
& S^{\prime}(x, y)=\lambda(x, y) S^{1}(x, y)+\mu(x, y) S^{2}(x, y), \\
& T^{\prime}(x, y)=\lambda(x, y) T^{1}(x, y)+\mu(x, y) T^{2}(x, y), \\
& A=\left[G r\left(R^{1}\right) \backslash G_{2}\right] \cup\left[G r\left(R^{2}\right) \backslash G_{1}\right], \\
& B=\left[G r\left(Q^{1}\right) \backslash G_{2}\right] \cup\left[G r\left(Q^{2}\right) \backslash G_{1}\right], \\
& R^{\prime}(x, y, u) \text { holds if and only if }(x, y, u) \in A, \\
& Q^{\prime}(x, y, v) \text { holds if and only if }(x, y, v) \in B,
\end{aligned}
$$

where

$$
\begin{array}{ll}
\lambda(x, y)=\frac{d\left((x, y), \bar{W}_{2}\right)}{d\left((x, y), \bar{W}_{1}\right)+d\left((x, y), \bar{W}_{2}\right)}, & \forall(x, y) \in X \times Y, \\
\mu(x, y)=\frac{d\left((x, y), \bar{W}_{1}\right)}{d\left((x, y), \bar{W}_{1}\right)+d\left((x, y), \bar{W}_{2}\right)}, & \forall(x, y) \in X \times Y .
\end{array}
$$

Easily, we can check that

(i) $S^{\prime}, T^{\prime}$ are continuous with nonempty compact convex values.

(ii) Since $\operatorname{Gr}\left(R^{1}\right)$ and $\operatorname{Gr}\left(R^{2}\right)$ are closed in $X \times Y \times X, A$ is closed in $X \times Y \times X$, which implies that $R^{\prime}(\cdot, \cdot, \cdot)$ is closed. Similarly, $Q^{\prime}(\cdot, \cdot, \cdot)$ is closed.

(iii) Suppose that there exist $y \in Y$, a finite subset $\left\{x^{1}, \ldots, x^{n}\right\} \subset X$ and $x \in \operatorname{co}\left\{x^{1}, \ldots, x^{n}\right\}$ such that $R^{\prime}\left(x, y, x^{i}\right)$ does not hold for any $i \in\{1, \ldots, n\}$, then

$$
\left(x, y, x^{i}\right) \notin A=\left[G r\left(R^{1}\right) \backslash G_{2}\right] \cup\left[G r\left(R^{2}\right) \backslash G_{1}\right], \quad \forall i \in\{1, \ldots, n\} .
$$

As $W_{1} \cap W_{2}=\emptyset$, without loss of generality, we may assume that $(x, y) \in(X, Y) \backslash W_{1}$. Therefore,

$$
\left(x, y, x^{i}\right) \notin G r\left(R^{2}\right) \backslash G_{1}, \quad \forall i \in\{1, \ldots, n\},
$$

i.e.,

$$
\left(x, y, x^{i}\right) \notin \operatorname{Gr}\left(R^{2}\right) \cap\left[(X, Y) \backslash W_{1} \times X\right], \quad \forall i \in\{1, \ldots, n\},
$$

which implies that $\left(x, y, x^{i}\right) \in G r\left(R^{2}\right)$ for any $i \in\{1, \ldots, n\}$. Thus $R^{2}\left(x, y, x^{i}\right)$ does not hold for any $i \in\{1, \ldots, n\}$, which is a contradiction. Hence, for any $y \in Y$, any finite subset $\left\{x^{1}, \ldots, x^{n}\right\}$ of $X$ and any $x \in \operatorname{co}\left\{x^{1}, \ldots, x^{n}\right\}$, there is $i \in\{1, \ldots, n\}$ such that $R^{\prime}\left(x, y, x^{i}\right)$ holds. Similarly, for any $x \in Y$, any finite subset $\left\{y^{1}, \ldots, y^{n}\right\}$ of $Y$ and any $y \in c o\left\{y^{1}, \ldots, y^{n}\right\}$, there is $i \in\{1, \ldots, n\}$ such that $Q^{\prime}\left(x, y, y^{i}\right)$ holds. Hence $q^{\prime} \in \mathcal{M}$. 
(iv) Further, by Lemma 3.3 and Lemma 3.4, we have

$$
\begin{aligned}
& \sup _{(x, y) \in X \times Y} h_{X}\left(S(x, y), S^{\prime}(x, y)\right) \leq \sup _{(x, y) \in X \times Y} h_{X}\left(S(x, y), S^{1}(x, y)\right) \\
& +\sup _{(x, y) \in X \times Y} h_{X}\left(S^{1}(x, y), S^{\prime}(x, y)\right) \\
& \leq \frac{\delta^{*}}{32}+\frac{\delta^{*}}{16} \\
& \leq \frac{3}{32} \delta^{*} \\
& \sup _{(x, y) \in X \times Y} h_{Y}\left(T(x, y), T^{\prime}(x, y)\right) \leq \sup _{(x, y) \in X \times Y} h_{Y}\left(T(x, y), T^{1}(x, y)\right) \\
& +\sup _{(x, y) \in X \times Y} h_{Y}\left(T^{1}(x, y), T^{\prime}(x, y)\right) \\
& \leq \frac{\delta^{*}}{32}+\frac{\delta^{*}}{16} \\
& \leq \frac{3}{32} \delta^{*} \\
& h\left(\operatorname{Gr}(R), \operatorname{Gr}\left(R^{\prime}\right)\right) \leq h\left(\operatorname{Gr}(R), \operatorname{Gr}\left(R^{1}\right)\right)+h\left(\operatorname{Gr}\left(R^{1}\right), \operatorname{Gr}\left(R^{\prime}\right)\right) \\
& \leq \frac{\delta^{*}}{32}+\frac{\delta^{*}}{16} \\
& \leq \frac{3}{32} \delta^{*} \\
& h^{\prime}\left(\operatorname{Gr}(Q), G r\left(Q^{\prime}\right)\right) \leq h^{\prime}\left(\operatorname{Gr}(Q), G r\left(Q^{1}\right)\right)+h^{\prime}\left(\operatorname{Gr}\left(Q^{1}\right), \operatorname{Gr}\left(Q^{\prime}\right)\right) \\
& \leq \frac{\delta^{*}}{32}+\frac{\delta^{*}}{16} \\
& \leq \frac{3}{32} \delta^{*} \text {. }
\end{aligned}
$$

Hence

$$
\begin{aligned}
\rho\left(q^{\prime}, q\right)= & \sup _{(x, y) \in X \times Y} h_{X}\left(S(x, y), S^{\prime}(x, y)\right)+\sup _{(x, y) \in X \times Y} h_{Y}\left(T(x, y), T^{\prime}(x, y)\right) \\
& +h\left(\operatorname{Gr}(R), G r\left(R^{\prime}\right)\right)+h^{\prime}\left(\operatorname{Gr}(Q), \operatorname{Gr}\left(Q^{\prime}\right)\right) \\
\leq & \frac{3}{8} \delta^{*} \\
< & \delta^{*} .
\end{aligned}
$$

Thus $q^{\prime} \in \mathcal{M}$ and $\rho\left(q^{\prime}, q\right)<\delta^{*}$.

Since

$$
\left(\Lambda\left(q^{\prime}\right) \cap W_{1}\right) \cup\left(\Lambda\left(q^{\prime}\right) \cap W_{2}\right)=\Lambda\left(q^{\prime}\right) \cap\left(W_{1} \cup W_{2}\right) \neq \emptyset,
$$

we assume $\Lambda\left(q^{\prime}\right) \cap W_{1} \neq \emptyset$ without loss of generality. Then there exists $(\bar{x}, \bar{y}) \in \Lambda\left(q^{\prime}\right) \cap W_{1}$ such that $(\bar{x}, \bar{y}) \in W_{1}, \bar{x} \in S^{\prime}(\bar{x}, \bar{y}), \bar{y} \in T^{\prime}(\bar{x}, \bar{y})$, and $R^{\prime}(\bar{x}, \bar{y}, u)$ and $Q^{\prime}(\bar{x}, \bar{y}, v)$ hold for any $u \in$ 
$S^{\prime}(\bar{x}, \bar{y})$ and any $v \in T^{\prime}(\bar{x}, \bar{y})$. Since $(\bar{x}, \bar{y}) \in W_{1}$,

$$
\begin{aligned}
& S^{\prime}(\bar{x}, \bar{y})=S^{1}(\bar{x}, \bar{y}), \quad T^{\prime}(\bar{x}, \bar{y})=T^{1}(\bar{x}, \bar{y}), \\
& R^{1}(\bar{x}, \bar{y}, u) \quad \text { and } \quad Q^{1}(\bar{x}, \bar{y}, v) \text { hold } \forall u \in S^{1}(\bar{x}, \bar{y}), \forall v \in T^{1}(\bar{x}, \bar{y}),
\end{aligned}
$$

which implies that $(\bar{x}, \bar{y}) \in \Lambda\left(q^{1}\right)$. Hence $\Lambda\left(q^{1}\right) \cap W_{1} \neq \emptyset$, which contradicts $\Lambda\left(q^{1}\right) \cap W_{1}=\emptyset$. Thus $m(q)$ is connected.

Theorem 3.6 For each $q \in \mathcal{M}$, there exists at least one essential component of $\Lambda(q)$.

Proof By Theorem 3.5, there exists at least one connected minimal essential subset $m(q)$ of $\Lambda(q)$. Thus, there is a component $C$ of $\Lambda(q)$ such that $m(q) \subset C$. It is obvious that $C$ is essential by Remark 3.1(2). Thus $C$ is an essential component.

Remark 3.2 Our paper has improved the results of [5]. (i) The symmetric (vector) quasiequilibrium problem is a special case of symmetric variational relation problem; (ii) In [5], the existence of essential connected components is based on disturbance of $S, T$ for fixed $f_{0}, g_{0}$ (see Section 4 in [5]), but the existence of essential connected components in our paper is based on disturbance of $S, T, R, Q$, which are more general.

\section{Conclusion}

In this paper, symmetric variational relation problems are introduced, and we establish the existence theorem of solutions of symmetric variational relation problems. Further, we study the notion of essential stability of equilibria of symmetric variational relation problems. Our paper improves the results of [5].

\section{Competing interests}

The author declares that they have no competing interests.

\section{Author's contributions}

The author read and approved the final manuscript.

\section{Acknowledgements}

Supported by the open project of Key Laboratory of Mathematical Economics (SUFE), Ministry of Education (Project Number: 201309KF02)

Received: 25 July 2013 Accepted: 1 December 2013 Published: 02 Jan 2014

\section{References}

1. Noor, MA, Oettli, W: On general nonlinear complementarity problems and quasi-equilibria. Matematiche XLIX, 313-331 (1994)

2. Blum, E, Oettli, W: From optimization and variational inequalities to equilibrium problems. Math. Stud. 63, 123-145 (1994)

3. Fu, JY: Symmetric vector quasi-equilibrium problems. J. Math. Anal. Appl. 285, 708-713 (2003)

4. Farajzadeh, AP: On the symmetric vector quasi-equilibrium problems. J. Math. Anal. Appl. 322, 1099-1110 (2006)

5. Chen, JC, Gong, XH: The stability of set of solutions for symmetric vector quasi-equilibrium problems. J. Optim. Theory Appl. 136(3), 359-374 (2008)

6. Zhang, WY: Well-posedness for convex symmetric vector quasi-equilibrium problems. J. Math. Anal. Appl. 387(2), 909-915 (2012)

7. Long, XJ, Huang, NJ: Metric characterizations of $\alpha$-well-posedness for symmetric quasi-equilibrium problems. J. Glob. Optim. 45(3), 459-471 (2009)

8. Fakhar, M, Zafarani, J: Generalized symmetric vector quasiequilibrium problems. J. Optim. Theory Appl. 136, 397-409 (2008)

9. Luc, DT: An abstract problem in variational analysis. J. Optim. Theory Appl. 138, 65-76 (2008)

10. Khanh, PQ, Luc, DT: Stability of solutions in parametric variational relation problems. Set-Valued Var. Anal. 16(7-8), 1015-1035 (2008) 
11. Pu, YJ, Yang, Z: Stability of solutions for variational relation problems with applications. Nonlinear Anal. 75, 1758-1767 (2012)

12. Lin, LJ, Wang, SY: Simultaneous variational relation problems and related applications. Comput. Math. Appl. 58 1711-1721 (2009)

13. Balaj, M, Luc, DT: On mixed variational relation problems. Comput. Math. Appl. 60, 2712-2722 (2010)

14. Balaj, M, Lin, LJ: Equivalent forms of a generalized KKM theorem and their applications. Nonlinear Anal. 73, 673-682 (2010)

15. Balaj, M, Lin, LJ: Generalized variational relation problems with applications. J. Optim. Theory Appl. 148, 1-13 (2011)

16. Lin, LJ, Ansari, QH: Systems of quasi-variational relations with applications. Nonlinear Anal. 72, 1210-1220 (2010)

17. Agarwal, RP, Balaj, M, O'Regan, D: A unifying approach to variational relation problems. J. Optim. Theory Appl. 155 417-429 (2012)

18. Balaj, M, Lin, LJ: Existence criteria for the solutions of two types of variational relation problems. J. Optim. Theory Appl. $156,232-246(2013)$

19. Luc, DT, Sarabi, E, Soubeyran, A: Existence of solutions in variational relation problems without convexity. J. Math. Anal. Appl. 364, 544-555 (2010)

20. Pu, YJ, Yang, Z: Variational relation problem without the KKM property with applications. J. Math. Anal. Appl. 393, 256-264 (2012)

21. Yang, Z, Pu, YJ: Generalized Knaster-Kuratowski-Mazurkiewicz theorem without convex hull. J. Optim. Theory Appl. 154(1), 17-29 (2012)

22. Yang, Z, Pu, YJ: Existence and stability of solutions for maximal element theorem on Hadamard manifolds with applications. Nonlinear Anal. 75(2), 516-525 (2012)

23. Aubin, JP, Ekeland, I: Applied Nonlinear Analysis. Wiley, New York (1984)

24. Fort, MK Jr.: A unified theory of semi-continuity. Duke Math. J. 16(2), 237-246 (1949)

25. Yu, J, Luo, Q: On essential components of the solution set of generalized games. J. Math. Anal. Appl. 230, 303-310 (1999)

26. $\mathrm{Yu}, \mathrm{J}, \mathrm{Zhou}, \mathrm{YH}$ : A Hausdorff metric inequality with applications to the existence of essential components. Nonlinear Anal. 69, 1851-1855 (2008)

10.1186/1029-242X-2014-5

Cite this article as: Yang: On existence and essential stability of solutions of symmetric variational relation problems. Journal of Inequalities and Applications 2014, 2014:5

\section{Submit your manuscript to a SpringerOpen ${ }^{\circ}$ journal and benefit from:}

- Convenient online submission

Rigorous peer review

- Immediate publication on acceptance

- Open access: articles freely available online

- High visibility within the field

- Retaining the copyright to your article 